\title{
Caracterização de farinhas de tapioca produzidas no estado do Pará
}

\author{
Characterization of tapioca flour obtained in Pará state, Brazil \\ Priscilla Andrade Silva' Roberto Lisboa Cunha" Alessandra Santos Lopes ${ }^{\text {III }}$ \\ Rosinelson da Silva Pena ${ }^{\text {III* }}$
}

RESUMO

\begin{abstract}
A farinha de tapioca é um alimento produzido artesanalmente a partir da fécula de mandioca (Manihot esculenta Crantz), amplamente consumida na Região Amazônica. $O$ objetivo deste trabalho foi caracterizar duas farinhas de tapioca produzidas no estado do Pará: uma no Baixo Amazonas e outra na Zona Bragantina. As duas farinhas apresentaram perfis granulométricos distintos e diferença significativa $(P<0,05)$ para a maioria dos parâmetros físicoquímicos e tecnológicos analisados. A farinha de tapioca proveniente do Baixo Amazonas apresentou maior umidade (10,7\%), em função da maior capacidade de adsorver água, devido a sua maior área específica (menor granulometria). A microscopia óptica com luz polarizada, juntamente com as características dos dois produtos indicou a inexistência de um processo padrão utilizado na produção da farinha de tapioca.

Palavras-chave: Manihot esculenta, farinha, produto amiláceo, propriedades, microscopia.
\end{abstract}

\section{ABSTRACT}

Tapioca flour is a typical food produced from cassava starch (Manihot esculenta Crantz), widely consumed in the Amazon Region. The aim of this study was to characterize two tapioca flours produced in Pará State, Brazil one in the West Region and other in Bragantina Area. Both tapioca flour presented distinct particle-size distribution and significant difference $(P<0.05)$ for majority of the physico-chemical and technological parameters. Flour from West Region presented high moisture (10.7\%), this due the greater capacity of water adsorption, attributed at its high specific area (smaller particlesize). The optical microscopy with polarized light and the technologic properties of the products did not showed a standard process for the production of tapioca flour.

Key words: Manihot esculenta, flour, starchy food, properties, microscopy.

\section{INTRODUÇÃO}

A mandioca (Manihot esculenta Crantz) se destaca como uma das principais culturas do Brasil, e a maior parte da sua produção é destinada à fabricação da farinha de mandioca. O restante é utilizado na alimentação humana e animal e na obtenção da fécula (BELEIA et al., 2006; FRANCK et al., 2011). A fécula é a forma mais ampla de aproveitamento industrial da mandioca e é empregada como matéria-prima no processamento de diversos alimentos (NWOKOCHA et al., 2009; CARVALHO et al., 2010).

Em muitos países, as denominações cassava starch, tapioca flour e tapioca starch são confundidas com a denominação farinha de tapioca, mas significam fécula de mandioca, que é um produto diferente (MILDE et al., 2010; POONGODI VIJAYAKUMAR \& BOOPATHY, 2012). A farinha de tapioca é um produto característico das regiões Norte e Nordeste do Brasil, que é produzido a partir da fécula extraída das raízes da mandioca (CHISTÉ et al., 2012).

'Programa de Pós-graduação em Ciência e Tecnologia de Alimentos, Instituto de Tecnologia, Universidade Federal do Pará (UFPA), Belém, PA, Brasil.

"Embrapa Amazônia Oriental, Belém, PA, Brasil.

"'Faculdade de Engenharia de Alimentos, Instituto de Tecnologia, UFPA, Rua Augusto Corrêa, 01, Guamá, 66075-900, Belém, PA, Brasil. E-mail: rspena@ufpa.br. *Autor para correspondência. 
Da mesma forma que outros derivados da mandioca, a farinha de tapioca apresenta elevado teor de amido e baixo teor de proteínas, lipídios e minerais, o que faz dela um alimento altamente calórico (CHISTÉ et al., 2012). O produto é consumido na região amazônica de diferentes maneiras e é comercializado principalmente em feiras livres. Segundo informação de produtores, na Zona Bragantina estão localizadas as casas de farinha de tapioca do estado do Pará, onde o produto é elaborado de forma artesanal e em pequena escala. A Comunidade de Americano, no município de Santa Izabel, é a principal produtora.

$\mathrm{O}$ regulamento técnico de identidade e qualidade dos produtos amiláceos derivados da raiz de mandioca (BRASIL, 2005) estabelece os limites de tolerância para os produtos amiláceos derivados da raiz de mandioca e define a farinha de tapioca como o produto que, conforme processo de fabricação, apresenta-se sob forma de grânulos irregulares, poliédricos ou esféricos.

O objetivo do trabalho foi avaliar propriedades físicas, físico-químicas, tecnológicas e morfológicas de duas farinhas de tapioca com características físicas diferentes, produzidas no estado do Pará, como uma contribuição para a padronização do processo de produção do produto.

\section{MATERIAL E MÉTODOS}

Material

No estudo, foram utilizadas duas farinhas de tapioca, adquiridas diretamente dos produtores, em feiras livres das cidades de Santarém e de Belém, ambos municípios do estado do Pará. A primeira farinha foi produzida no Baixo Amazonas (município de Santarém) e a segunda na Zona Bragantina (município de Santa Izabel).

De acordo com o produtor de Santa Izabel, na obtenção do produto, a fécula de mandioca umedecida é passada por peneira, malha 3,0mm. As partículas passantes são pressionadas sobre um tecido de algodão emoldurado, com movimento leve e giratório das mãos, para formar grânulos, os quais são peneirados novamente na mesma malha. Segue-se então para o escaldamento, sobre a chapa do forno a aproximadamente $180^{\circ} \mathrm{C}$, com revolvimento constantemente, por um tempo curto $(<5 \mathrm{~min})$. Em seguida, os grânulos ficam em repouso por 24 horas à temperatura ambiente e são submetidos à espocagem, no forno a $240^{\circ} \mathrm{C}$, também com constantemente revolvimento; os grânulos saltam como pipoca e a operação é finalizada quando cessa esse movimento. Segundo o produtor de Santarém, no processamento da sua farinha, a etapa de escaldamento é suprimida, sendo os grânulos submetidos diretamente à espocagem a $240^{\circ} \mathrm{C}$. O primeiro produto é mais leve e expandido que o segundo.

Caracterização física e físico-química das farinhas de tapioca

Foram realizadas as análises de: Umidadepor gravimetria, método 920.151 da AOAC (1997); $p H-$ por potenciometria, método 981.12 da AOAC (1997); Acidez total titulável - pela metodologia 02-31 da AACC (1983); Atividade de água $\left(a_{w}\right)$ - por leitura direta em termohigrômetro digital; Proteínas totais por Kjeldahl, método 920.87 da AOAC (1997), com fator nitrogênio-proteína de 5,75 (proteínas vegetais) (BRASIL, 2003); Lipídios - pelo método 922.06 da AOAC (1997); Cinzas - pelo método 930.05 da AOAC (1997); Amido total - conforme metodologia descrita por CEREDA et al. (2004); Carboidratos totais calculado por diferença, segundo BRASIL (2003); Cor instrumental - por colorimetria tristimulus, pelo sistema CIE Lab; Granulometria - de acordo com o método 965.22 da AOAC (1997), com um conjunto de peneiras (4, 6, 9 e 20 mesh e um fundo-ABNT), em classificador vibratório marca Produtest; Valor calórico - de acordo com BRASIL (2003).

Caracterização tecnológica das farinhas de tapioca Índice de absorção de água (IAA) e Índice de solubilidade em água (ISA) - A determinação dos parâmetros foi feita de acordo com a metodologia proposta por ANDERSON et al. (1969).

Densidade aparente - Determinada pelo método do deslocamento da massa ocupada (p), utilizando sementes de canola. Foi medido o volume (v) deslocado em proveta graduada e determinada à densidade aparente das farinhas $(\rho=\mathrm{p} / \mathrm{v})$ (RAMIREZ \&WANDERLEY, 1997).

Higroscopicidade das farinhas - Nessa análise, aproximadamente $1 \mathrm{~g}$ da farinha foi pesado em cápsula de polipropileno, e colocado em recipiente hermético, contendo solução saturada de $\mathrm{NaCl}$ (umidade relativa de $75 \%$ ). A higroscopicidade é definida como a umidade de equilíbrio da farinha, quando expostas ao referido ambiente a $25^{\circ} \mathrm{C}$ (TONON et al., 2009).

\section{Avaliação morfológica das farinhas}

A caracterização morfológica dos grânulos de amido das farinhas foi realizada em microscópio eletrônico de varredura marca LEO, modelo 1450VP. Foi também avaliado o padrão de birrefringência do amido, em microscópio ótico de luz polarizada marca Leica, modelo DM/LP, com câmera digital DC300F acoplada. 
Análise estatística

As análises físico-químicas e tecnológicas das farinhas foram realizadas em triplicata e os resultados foram avaliados através de análise de variância (ANOVA) e teste complementar de comparação de médias de Tukey, com auxílio do programa STATISTICA Kernel Release 7.1 (StatSoft Inc., 2006, Tulsa, OK, USA).

\section{RESULTADOS E DISCUSSÃO}

Caracterização física e físico-química das farinhas de tapioca

Os resultados da análise granulométrica e da caracterização físico-química das farinhas de tapioca estão nas tabelas 1 e 2 , respectivamente. De acordo com a análise granulométrica, enquanto a maior fração da farinha de tapioca de Santa Izabel ( $\approx 98 \%$ ) apresentou tamanho de partícula superior a $3,35 \mathrm{~mm}$, para a farinha de Santarém ( $\approx 90 \%)$, a maior fração apresentou tamanho inferior a 3,35mm. Os resultados indicam que não existe um padrão granulométrico para o produto, e que provavelmente a etapa de escaldamento, a qual é submetida a farinha de Santa Izabel, favorece a expansão dos grânulos. Essa etapa favorece a formação de uma camada superficial de amido gelatinizado nos grânulos, que proporciona o aumento da pressão no seu interior durante a espocagem, e a obtenção de grânulos com maior volume.

De acordo com a tabela 2 , a atividade de água $\left(\mathrm{a}_{\mathrm{w}}\right)$ das duas farinhas $(0,18$ e 0,46$)$ permite afirmar que elas apresentaram estabilidade microbiológica $\left(a_{\mathrm{w}}<0,6\right)$ (JAY, 2005), embora os produtos sejam classificados como de baixa acidez, por apresentarem pH maior que 4,5. A acidez dos produtos (média 0,77\%) atendeu a Legislação Brasileira, que permite um máximo de 2,0\% para o produto (BRASIL, 2005), e são da ordem de grandeza observada por DIAS \& LEONEL (2006) (média 0,94\%), em farinhas de mandioca de diferentes procedências, e por LADEIRA \& PENA (2011) (0,5$1,0 \%)$, em féculas de mandioca.

Além do padrão de acidez, as farinhas também atenderam aos padrões de umidade $(<15 \%) \mathrm{e}$ cinzas $(<0,5 \%)$, estabelecidos pela Legislação Brasileira para o produto (BRASIL, 2005). De maneira geral, a composição das farinhas de tapioca estão de acordo com a composição observada por CHISTÉ et al. (2006), para farinhas de mandioca tipo seca: umidade $(5,48-$ $7,59 \%), a_{w}(0,31-0,61)$, cinzas $(0,54-0,90 \%)$, lipídios $(0,11-$ $0,31 \%)$ e proteínas $(0,53-0,93 \%)$; exceto o amido $(67,67$ $79,59 \%$ ), que foi inferior.

A farinha de Santarém apresentou maior umidade e $\mathrm{a}_{\mathrm{w}}$, o que pode ser atribuído à maior capacidade de adsorver água, devido a sua maior área específica (menor granulometria). Por outro lado, o teor de amido dessa farinha foi inferior, o que pode ser atribuído à pureza da fécula utilizada. $\mathrm{O}$ parâmetro de cor $b^{*}$ foi estatisticamente superior $(\mathrm{P}<0,05)$ para a farinha de Santarém, indicando ser um produto suavemente mais amarelado, o que pode também ser atribuído à qualidade da fécula.

O constituinte majoritário das duas farinhas foi os carboidratos, que representaram 88,23 e 94,49\% das farinhas de Santarém e de Santa Izabel, respectivamente. Os carboidratos são as maiores fontes energéticas nos alimentos e, no caso das farinhas estudadas, são responsáveis por fornecer 361,16 e 386,02kcal 100 g-1 , para as farinhas de Santarém e de Santa Izabel, respectivamente. Os valores são próximos ao reportado por TACO (2006) (331 kcal 100g $\mathrm{g}^{-1}$ ) para o produto.

Caracterização tecnológica das farinhas

Na tabela 2, são apresentados também os resultados dos parâmetros tecnológicos índice de absorção de água (IAA) e índice de solubilidade em

Tabela 1 - Análise granulométrica das farinhas de tapioca de Santarém e de Santa Izabel.

\begin{tabular}{|c|c|c|c|c|c|}
\hline \multirow{2}{*}{ Procedência da farinha } & \multicolumn{5}{|c|}{ Percentagem retida por tamanho de partícula $(m e s h)^{1}$} \\
\hline & $>4$ & $4-6$ & $6-9$ & $9-20$ & $<20$ \\
\hline Santarém $(\%)$ & $0,84 \pm 0,05^{\mathrm{b}}$ & $9,59 \pm 0,45^{\mathrm{b}}$ & $64,82 \pm 0,54^{\mathrm{a}}$ & $14,86 \pm 0,14^{\mathrm{a}}$ & $9,88 \pm 0,16^{2}$ \\
\hline Santa Izabel (\%) & $4,33 \pm 0,11^{\mathrm{a}}$ & $93,63 \pm 0,51^{\mathrm{a}}$ & $1,90 \pm 0,32^{\mathrm{b}}$ & $0,03 \pm 0,01^{\mathrm{b}}$ & $0,10 \pm 0,10^{\mathrm{b}}$ \\
\hline
\end{tabular}

Ciência Rural, v.43, n.1, jan, 2013. 
Tabela 2 - Composição e propriedades físico-químicas e tecnológicas das farinhas de tapioca de Santarém e de Santa Izabel.

\begin{tabular}{|c|c|c|}
\hline \multirow{2}{*}{ Parâmetro ${ }^{1}$} & , n & - \\
\hline & Santarém & Santa Izabel \\
\hline Atividade de água $\left(\mathrm{a}_{\mathrm{w}}\right)$ & $0,46 \pm 0,01^{\mathrm{a}}$ & $0,18 \pm 0,02^{\mathrm{b}}$ \\
\hline Acidez titulável (\%) & $0,76 \pm 0,06^{\mathrm{a}}$ & $0,78 \pm 0,05^{\mathrm{a}}$ \\
\hline $\mathrm{pH}$ & $5,75 \pm 0,01^{\mathrm{a}}$ & $5,29 \pm 0,02^{\mathrm{b}}$ \\
\hline Umidade (\%) & $10,69 \pm 0,12^{\mathrm{a}}$ & $4,53 \pm 0,05^{\mathrm{b}}$ \\
\hline Proteínas (\%) & $0,08 \pm 0,03^{\mathrm{a}}$ & $0,08 \pm 0,05^{\mathrm{a}}$ \\
\hline Lipídios (\%) & $0,88 \pm 0,18^{\mathrm{a}}$ & $0,86 \pm 0,48^{\mathrm{a}}$ \\
\hline Cinzas (\%) & $0,12 \pm 0,04^{\mathrm{a}}$ & $0,04 \pm 0,02^{\mathrm{b}}$ \\
\hline Amido $(\%)$ & $84,30 \pm 1,78^{b}$ & $93,03 \pm 0,64^{\mathrm{a}}$ \\
\hline \multirow{3}{*}{$\begin{array}{ll}\text { Cor } & L^{*} \\
& a^{*} \\
& b^{*}\end{array}$} & $89,45 \pm 0,10^{\mathrm{a}}$ & $88,54 \pm 0,40^{b}$ \\
\hline & $0,04 \pm 0,02^{\mathrm{b}}$ & $0,20 \pm 0,01^{\mathrm{a}}$ \\
\hline & $4,49 \pm 0,11^{\mathrm{a}}$ & $3,81 \pm 0,14^{\mathrm{b}}$ \\
\hline Carboidratos totais (\%) & $88,23 \pm 0,06^{\mathrm{b}}$ & $94,49 \pm 0,17^{\mathrm{a}}$ \\
\hline VET (kcal/100g) & $361,16 \pm 1,98^{\mathrm{b}}$ & $386,02 \pm 5,20^{\mathrm{a}}$ \\
\hline IAA (g de gel/g MS) & $7,43 \pm 0,08^{\mathrm{a}}$ & $6,53 \pm 0,03^{\mathrm{b}}$ \\
\hline $\operatorname{ISA}(\%)$ & $6,77 \pm 0,77^{\mathrm{b}}$ & $19,27 \pm 1,21^{\mathrm{a}}$ \\
\hline Densidade aparente $\left(\mathrm{g} / \mathrm{cm}^{3}\right)$ & $0,61 \pm 0,02^{\mathrm{a}}$ & $0,10 \pm 0,04^{\mathrm{b}}$ \\
\hline Higroscopicidade ( $\mathrm{g} \mathrm{H}_{2} \mathrm{O} / \mathrm{g} \mathrm{MS}$ ) & $0,58 \pm 0,01^{\mathrm{a}}$ & $0,17 \pm 0,02^{\mathrm{b}}$ \\
\hline
\end{tabular}

${ }^{1}$ Valores representam a média das triplicatas \pm desvio-padrão; VET - Valor energético total; MS - Matéria seca. Médias com letras iguais, na mesma linha, não diferem entre si pelo Teste de Tukey, a 5\% de probabilidade.

água (ISA), densidade aparente e higroscopicidade das farinhas de tapioca. Embora o IAA esteja relacionado com o grau de intumescimento ou gelatinização do amido, pois somente grânulos de amido gelatinizados conseguem absorver água em temperatura ambiente (MERCIER et al., 1998), é mais provável que o valor do IAA, estatisticamente superior $(\mathrm{P}<0,05)$ para a farinha de Santarém, seja atribuído a sua maior área superficial (menor tamanho de grânulos).

O ISA está relacionado com a quantidade de moléculas solúveis em água, como resultado da dextrinização do amido, e mede o grau de danificação do amido (MERCIER et al., 1998). O maior valor do ISA, altamente significativo $(\mathrm{P}<0,05)$ para a farinha de Santa Izabel, indica que os grânulos de amido do produto sofreram maiores danos em função do processo térmico utilizado, o que pode ser justificado pela sua maior expansão.

A densidade é um parâmetro importante na especificação de embalagem, transporte e armazenamento. $\mathrm{O}$ valor bem menor da densidade aparente da farinha de Santa Izabel $\left(0,10 \mathrm{~g} \mathrm{~cm}^{3}\right)$ já era esperado, por ser um produto bem mais expandido, devido ao processo de fabricação, como comprovou a análise granulométrica. A maior higroscopicidade da farinha de Santarém $\left(0,58 \mathrm{~g} \mathrm{H}_{2} \mathrm{O} \mathrm{g}^{-1} \mathrm{MS}\right)$ também pode ser atribuída a sua maior área superficial (menor granulometria), e indica que essa farinha requer melhores condições de acondicionamento e armazenamento, pois estará mais susceptível a perder sua estabilidade microbiológica $\left(a_{w}>0,6\right)$. Para o mesmo nível de umidade relativa (75\%), CHISTÉ et al. (2012) observaram umidade de equilíbrio de $0,17 \mathrm{~g} \mathrm{H}_{2} \mathrm{O} \mathrm{g}^{-1} \mathrm{MS}$ para farinha de tapioca; o mesmo valor observado para a farinha de Santa Izabel.

Caracterização morfológica das farinhas de tapioca

Nas eletromicrografias das farinhas (Figura 1), podem ser observadas modificações estruturais e a perda do formato esférico, característico dos grânulos de amido, o que é atribuído à gelatinização destes devido às elevadas temperaturas aplicadas no processamento do produto. Foi possível estimar o diâmetro dos grânulos de amido dos produtos, que, para a farinha de Santarém, variou de 10 a $20 \mu \mathrm{m}$ (média $15 \mu \mathrm{m})$, enquanto, para a farinha de Santa Izabel, a variação foi de 20 a $40 \mu \mathrm{m}$ (média $30 \mu \mathrm{m}$ ). Os valores permitem afirmar que houve maior inchamento dos grânulos do amido da farinha de Santa Izabel, em função do processo de fabricação.

As micrografias ópticas com luz polarizada (Figura 2) evidenciaram que a farinha de tapioca de Santa Izabel apresentou maior concentração de cruz de malta no interior dos grânulos, e que o amido da 


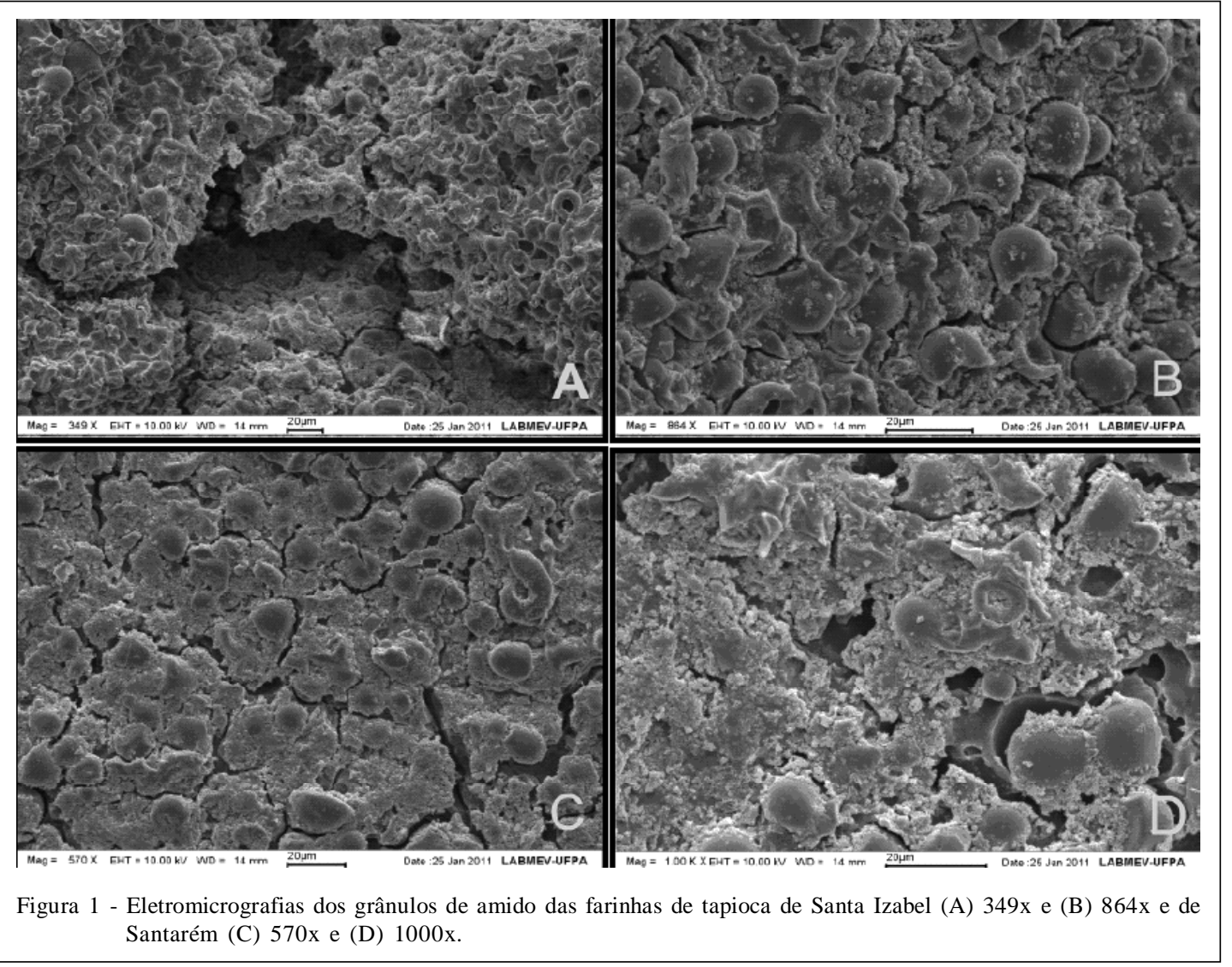

superfície sofreu gelatinização (ELIASSON, 2006). Por outro lado, os grânulos da farinha de Santarém apresentaram maior concentração de cruz de malta na superfície dos grânulos, indicando que foi o amido da parte mais central que sofreu gelatinização preferencial. O comportamento observado para a farinha de Santa Izabel é atribuído à etapa de escaldamento realizada antes da etapa de espocagem. $O$ fato de a temperatura de escaldamento $\left(\approx 180^{\circ} \mathrm{C}\right)$ ser inferior à temperatura de espocagem $\left(240^{\circ} \mathrm{C}\right)$ provoca uma redução na taxa de transferência de massa (perda de umidade) na superfície dos grânulos, permitindo a gelatinização do amido nessa posição. Já na farinha de Santarém, como os grânulos foram submetidos à etapa de espocagem a $240^{\circ} \mathrm{C}$ diretamente, as taxas de transferência de massa (perda de umidade) mais elevadas na superfície dos grânulos não permitiram que ocorresse a gelatinização do amido superficial; apenas do amido mais interno aos grânulos.

Por meio das micrografias óticas das farinhas é possível justificar o comportamento observado para a granulometria e a densidade das duas farinhas. A gelatinização do amido superficial dos grânulos da farinha de Santa Izabel favoreceu a formação de uma camada superficial impermeável, que impediu a difusão da água na etapa de espocagem, proporcionando uma maior expansão dos grânulos. Dessa forma, é possível justificar a maior granulometria e a menor densidade aparente dessa farinha.

\section{CONCLUSÃO}

As farinhas de tapioca produzidas em Santarém e Santa Izabel atenderam aos padrões da Legislação Brasileira, mas apresentaram propriedades físicas, físico-químicas, tecnológicas e morfológicas distintas. As diferenças altamente significativas entre as propriedades físicas, tecnológicas e morfológicas dos produtos confirmaram a inexistência de padronização no processo de produção da farinha de tapioca. A microscopia ótica comprovou que a etapa de escaldamento proporciona a formação de uma camada de amido gelatinizado na superfície dos grânulos, que favorece a sua expansão durante a espocagem. As propriedades analisadas são importantes parâmetros de processo e poderão ser utilizadas como subsídio para a padronização do processo de produção da farinha de tapioca. 


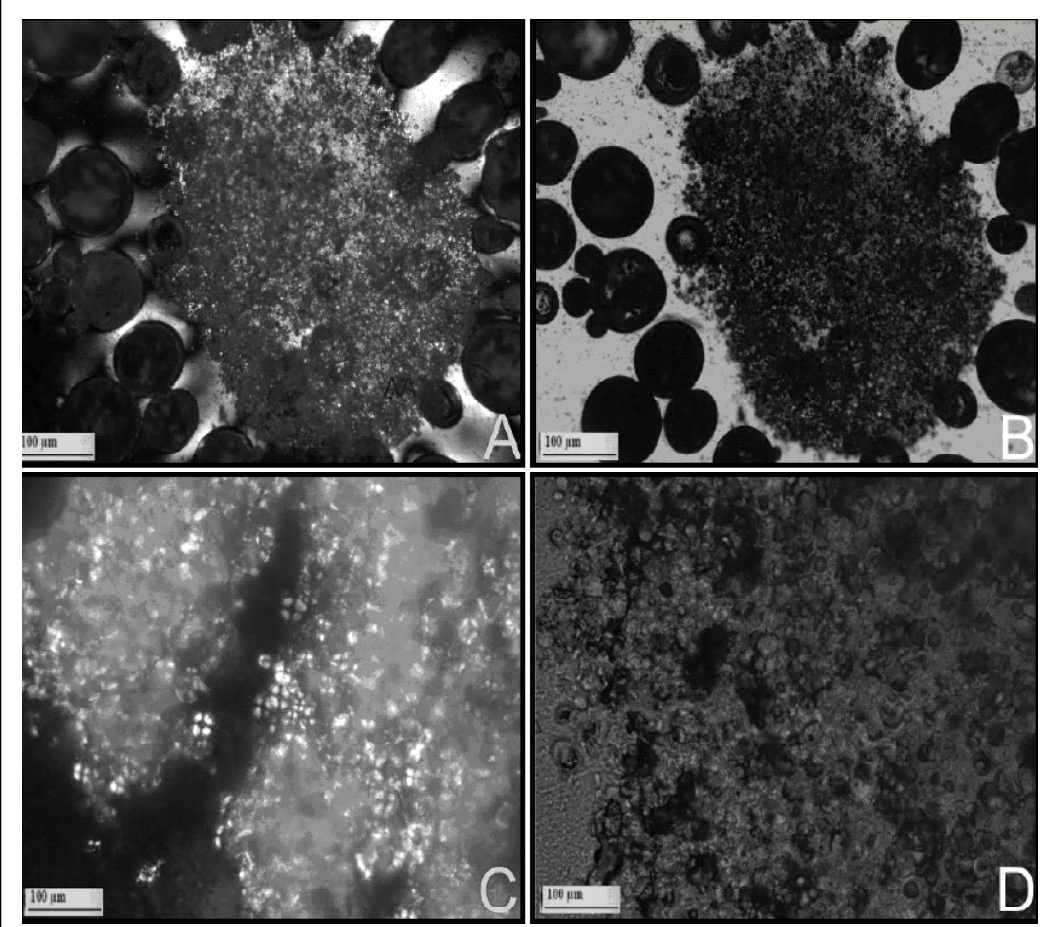

Figura 2 - Micrografias ópticas das farinhas de tapioca (Aumento: 40x). Santa Izabel: luz polarizada (A) e luz convencional (B); Santarém: luz polarizada (C) e luz convencional (D).

\section{AGRADECIMENTO}

Ao Conselho Nacional de Desenvolvimento Científico e Tecnológico (CNPq) e a Fundação de Amparo à Pesquisa do Estado do Pará (FAPESPA), pelo suporte financeiro.

\section{REFERÊNCIAS}

AACC (AMERICAN ASSOCIATION OF CEREAL CHEMISTS). Approved methods of the AACC. 8.ed. St. Paul, 1983. 1200p.

ANDERSON, R.A. et al. Gelatinization of corn grits by roll and extrusion cooking. Cereal Science Today, Saint. Paul, v.14, n.1, p.4-11, 1969.

AOAC (ASSOCIATION OF OFFICIAL ANALYTICAL CHEMISTS). Official methods of analysis of the AOAC international. 16.ed. 3.Rev. Washington, 1997. $1141 \mathrm{p}$.

BELEIA, A. et al. Modeling of starch gelatinization during cooking of cassava (Manihot esculenta Crantz). LWT - Food Science and Technology, London, v.39, n.4, p.400-405, 2006. Disponível em: <http://dx.doi.org/10.1016/j.lwt. 2005.02.021>. Acesso em: 16 ago. 2011. doi: 10.1016/ j.lwt.2005.02.021.

BRASIL. Agência Nacional de Vigilância Sanitária. Resolução RDC n.360, de 23 de dezembro de 2003. Regulamento Técnico sobre rotulagem nutricional de alimentos embalados. Diário
Oficial [da República Federativa do Brasil]. Brasília, dez. 2003. Seção 1, p.4.

BRASIL. Ministério da Agricultura, Pecuária e Abastecimento. Instrução Normativa n.23, de 14 de dezembro de 2005. Regulamento Técnico de Identidade e Qualidade dos Produtos Amiláceos derivados da raiz da mandioca. Diário Oficial [da República Federativa do Brasil], Brasília, dez. 2005. Seção 1, p.5.

CARVALHO, A.V. et al. Caracterização tecnológica de extrusado de terceira geração à base de farinhas de mandioca e pupunha. Ciência e Agrotecnologia, Lavras, v.34, n.4, p.995-1003, 2010. Disponível em: <http://www.editora.ufla.br/_adm/upload/ revista/34-42010_28.pdf>. Acesso em: 21 out. 2011.

CEREDA, M.P. et al. Metodologia de determinação de amido por digestão ácida em microondas. Revista da Associação Brasileira dos Produtores de Amido de Mandioca, Paranavaí, v.2, p.29, 2004. Disponível em: <http:// www.abam.com.br/revista/revista8/metodologia.php>. Acesso em: 18 maio, 2011.

CHISTÉ, R.C. et al. Qualidade da farinha de mandioca do grupo seca. Ciência e Tecnologia de Alimentos, Campinas, v.26, n.4, p.861-864, 2006. Disponível em: <http://www.scielo.br/ pdf/cta/v26n4/22.pdf>. Acesso em: 13 nov. 2011.

CHISTÉ, R.C. et al. Sorption isotherms of tapioca flour. International Journal of Food Science and Technology, Oxford, v.47, n.4, p.870-874, 2012. Disponível em: <http:// 
onlinelibrary.wiley.com.ez3.periodicos.capes.gov.br/doi/ 10.1111/j.13652621.2011.0xabstract>. Acesso em: 02 maio, 2012. doi: $10.1111 / \mathrm{j} .1365-2621.2011 .02900 . x$.

DIAS, L.T.; LEONEL, M. Caracterização físico-química de farinhas de mandioca de diferentes localidades do Brasil. Ciência e Agrotecnologia, Lavras, v.30, n.4, p.692-700, 2006. Disponível em: <http://www.editora.ufla.br/_adm/upload/ revista/30-4-2006_15.pdf>. Acesso em: 14 jul. 2011.

ELIASSON, A.C. (Ed.). Carbohydrates in food. 2.ed. Boca Raton: CRC, 2006. 563p.

FRANCK, H. et al. Effects of cultivar and harvesting conditions (age, season) on the texture and taste of boiled cassava roots. Food Chemistry, Amsterdan, v.126, n.1, p.127-133, 2011. Disponível em: 〈http://dx.doi.org/10.1016/j.foodchem.2010.10.088>. Acesso em: 23 abr. 2012. doi:10.1016/j.foodchem.2010.10.088.

JAY, M.J. Microbiologia de alimentos. 6.ed. Porto Alegre: Artmed, 2005. 711p.

LADEIRA, T.M.S.; PENA, R.S. Propriedades físico-químicas e tecnológicas dos polvilhos azedos de três cultivares de mandioca. Alimentos e Nutrição, Araraquara, v.22, n.4, p.631-640, 2011. Disponível em: <http://servbib.fcfar.unesp.br/seer/ index.php/alimentos/article/view/1654/1169>. Acesso em: 25 jan. 2012.

MERCIER, C. et al. Extrusion cooking. 2.ed. St. Paul: American Association of Cereal Chemists, 1998. 471p.

MILDE, B.L. et al. Gluten-free bread based on tapioca starch: texture and sensory studies. Food and Bioprocess Technology, Ireland, v.5, n.3, p.888-896, 2010. Disponível em: <http:// www.springerlink.com.ez3.periodicos.capes.gov.br/content/ t1013311278867gv/>. Acesso em: 08 maio, 2012. doi: 10.1007/s11947-010-0381-x.

NWOKOCHA, L.M. et al. A comparative study of some properties of cassava (Manihot esculenta Crantz) and cocoyam (Colocasia esculenta, Linn) starches. Carbohydrate Polymers, Barking, v.76, n.3, p.362-367, 2009. Disponível em: <http://dx.doi.org/10.1016/j.carbpol.2008.10.034>. Acesso em: 27 dez. 2011. doi: 10.1016/j.carbpol.2008.10.034.

POONGODI VIJAYAKUMAR, T.; BOOPATHY, P. Optimization of ingredients for noodle preparation using response surface methodology. Journal of Food Science and Technology, India, v.47, n.4, p.870-874, 2012. Disponível em: <http://www.springerlink.com.ez3.periodicos.capes.gov.br/ content/13814t0t24385145/>. Acesso em: 08 maio, 2012. doi: 10.1007/s13197-02-0641-z.

RAMIREZ, J.L.A.; WANDERLEY, C.P. Effect de los parametros de extrusion, caracteristicas de pasta y textura de pellets (snacks de terceira generacion) producidos a partir de trigo y maiz. Alimentaria, Madrid, v.279, n.1, p.93-98, 1997.

TACO. Tabela brasileira de composição de alimentos. 2.ed. Campinas: Universidade Estadual de Campinas, 2006. $113 \mathrm{p}$.

TONON, R.V. et al. Influência da temperatura do ar de secagem e da concentração de agente carreador sobre as propriedades físico-químicas do suco de açaí em pó. Ciência e Tecnologia de Alimentos, Campinas, v.29, n.2, p.444-450, 2009. Disponível em: 〈http://www.scielo.br/pdf/cta/v29n2/34.pdf >. Acesso em: 02 set. 2011. 